Supporting Information

\title{
Cationic $\pi$-Stacking Columns of Coronene Molecules with Fully Charged and Charge-Disproportionated States
}

\author{
Yukihiro Yoshida, ${ }^{\star \dagger}$ Kazuhide Isomura, ${ }^{\ddagger}$ Mitsuhiko Maesato, ${ }^{\S}$ Takashi Koretsune, ${ }^{\#, ~}$

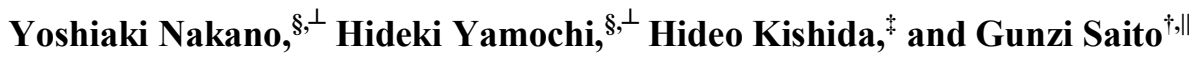

${ }^{\dagger}$ Faculty of Agriculture, Meijo University, Tempaku-ku, Nagoya 468-8502, Japan

$\$$ Department of Applied Physics, Nagoya University, Chikusa-ku, Nagoya 464-8603, Japan

$\S$ Division of Chemistry, Graduate School of Science, Kyoto University, Sakyo-ku, Kyoto 606-8502, Japan

\# Center for Emergent Matter Science, RIKEN, Wako 351-0198, Japan

ๆ JST, PRESTO, Saitama 332-0012, Japan

${ }^{\perp}$ Research Center for Low Temperature and Materials Sciences, Kyoto University, Sakyoku, Kyoto 606-8501, Japan

"| Toyota Physical and Chemical Research Institute, Nagakute 480-1192, Japan 
(a)

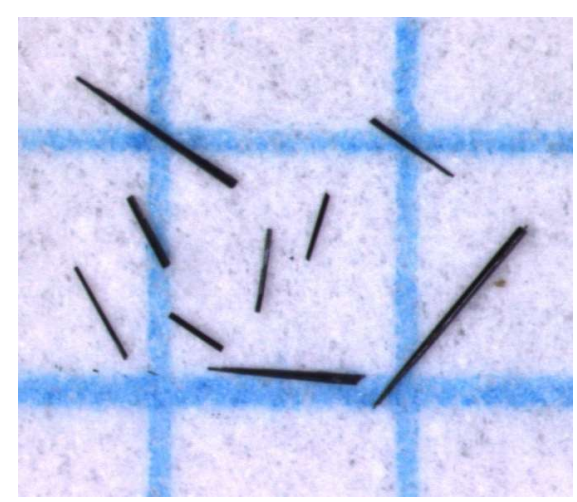

(b)

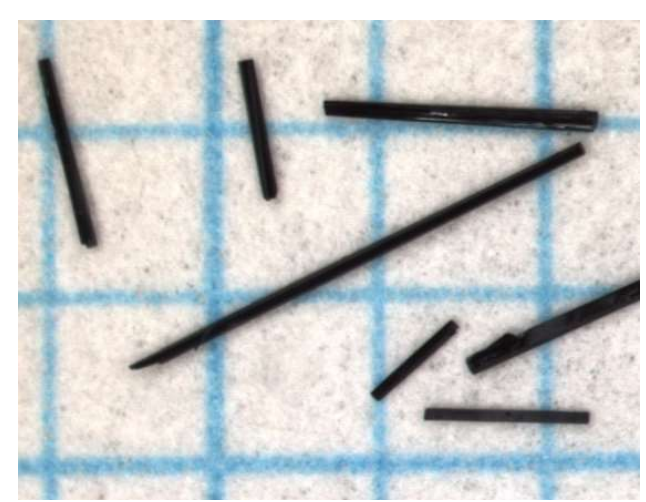

Figure S1. Photograph of rod-shaped crystals of (a) (coronene) $\left(\mathrm{GaCl}_{4}\right)$ (1) and (b) (coronene $)_{5}\left(\mathrm{GaCl}_{4}\right)_{2}(2)$ on a millimeter paper.

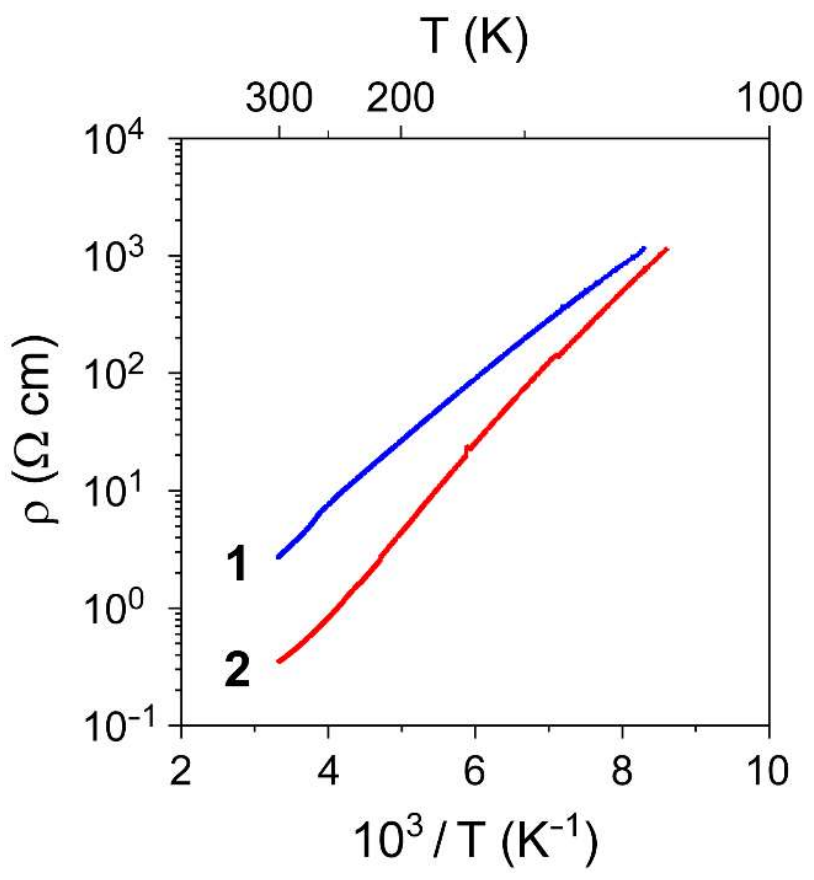

Figure S2. Logarithmic plot of the resistivity $(\rho)$ as a function of reciprocal temperature of a single crystal of $\mathbf{1}$ (blue) and $\mathbf{2}$ (red). 
Table S1. Intermolecular transfer integrals $(>20 \mathrm{meV})$ within the column for $\mathbf{1}$ calculated by first principles methods using the crystallographic data at $100 \mathrm{~K}$

\begin{tabular}{lc}
\hline Within a dimer & Transfer integral $(\mathrm{meV})$ \\
\hline$a_{\mathrm{u}}-a_{\mathrm{u}}$ & -120 \\
$b_{3 \mathrm{u}}-b_{3 \mathrm{u}}$ & -276 \\
\hline & \\
\hline Between dimers & Transfer integral $(\mathrm{meV})$ \\
\hline$a_{\mathrm{u}}-a_{\mathrm{u}}$ & 59 \\
$b_{3 \mathrm{u}}-b_{3 \mathrm{u}}$ & 135 \\
$a_{\mathrm{u}}-b_{3 \mathrm{u}}$ & -131 \\
\hline
\end{tabular}

Table S2. Point group, state, number of imaginary frequencies (NImag), total and relative energies $(E$ and $\Delta E)$, and expectation value of total spin angular momentum $\left(\left\langle S^{2}\right\rangle\right)$ of coronene $^{+}$at the UB3LYP/6-31+G(d,p) level of theory

\begin{tabular}{cccccc}
\hline Point group & State & NImag & $E$ (hartree) & $\Delta E(\mathrm{meV})$ & $\left\langle S^{2}\right\rangle$ \\
\hline$D_{2 \mathrm{~h}}$ & ${ }^{2} B_{3 u}$ & 2 & -921.68354 & 0.81 & 0.769 \\
$D_{2 \mathrm{~h}}$ & ${ }^{2} A_{u}$ & 1 & -921.68355 & 0.46 & 0.768 \\
$C_{2 \mathrm{~h}}$ & ${ }^{2} A_{u}$ & 1 & -921.68355 & 0.46 & 0.768 \\
$C_{\mathrm{i}}$ & ${ }^{2} A_{u}$ & 0 & -921.68357 & 0 & 0.768 \\
\hline
\end{tabular}


Table S3. Intermolecular transfer integrals $(>20 \mathrm{meV})$ within the column for 2 calculated by first principles methods using the crystallographic data at $100 \mathrm{~K}^{a}$

\begin{tabular}{lc}
\hline Between A-A & Transfer integral $(\mathrm{meV})$ \\
\hline$a_{\mathrm{u}}$-like $-a_{\mathrm{u}}$-like & 166 \\
\hline Between B-B & Transfer integral $(\mathrm{meV})$ \\
\hline$a_{\mathrm{u}}-a_{\mathrm{u}}$ & 62 \\
$b_{3 \mathrm{u}}-b_{3 \mathrm{u}}$ & -214 \\
\hline & \\
\hline Between $\mathbf{A}-\mathbf{B}$ & Transfer integral $(\mathrm{meV})$ \\
\hline$a_{\mathrm{u}}$-like $-a_{\mathrm{u}}$ & -50 \\
$a_{\mathrm{u}}-$ like $-b_{3 \mathrm{u}}$ & 206 \\
$b_{3 \mathrm{u}}$-like $-a_{\mathrm{u}}$ & -41 \\
$b_{3 \mathrm{u}}$-like $-b_{3 \mathrm{u}}$ & -47 \\
\hline
\end{tabular}

${ }^{a}$ Transfer integrals between the neighboring columns are at most about $20 \mathrm{meV}$. 